\title{
Los programas infantiles de televisión y su consumo en diferido en España
}

\section{Children's TV shows and their time shifted consumption in Spain}

\section{Programas de televisão para crianças e seu consumo diferido na Espanha}

\author{
Dr. Jorge Gallardo-Camacho \\ Director del Grado en Comunicación. Departamento de Comunicación \\ (Universidad Camilo José Cela) \\ http://orcid.org/0000-0003-3790-5105 \\ España \\ Dra. Eva Lavín \\ Profesora investigadora, departamento Comunicación Audiovisual \\ (Universidad Rey Juan Carlos) \\ http://orcid.org/0000-0002-8663-8632 \\ España

\section{Dr. Javier Sierra Sánchez} \\ Profesor Contratado Doctor \\ (Universidad Complutense de Madrid) \\ http://orcid.org/0000-0001-8572-7564 \\ España
}

Fecha de recepción: 15 de abril de 2019

Fecha de revisión: 26 de abril de 2019

Fecha de aceptación: 5 de diciembre de 2019

Fecha de publicación: 1 de enero de 2020

Para citar este artículo: Gallardo-Camacho, J., Lavín, E. y Sierra Sánchez, J. (2020). Los programas infantiles de televisión y su consumo en diferido en España, Icono 14, 18 (1), 155-178. doi: 10.7195/ri14.v18i1.1387 


\section{Resumen}

Este artículo presenta uno de los primeros estudios centrados en el consumo en diferido de los programas de televisión infantiles en España. La investigación analiza la cuantificación del peso de la audiencia en diferido en estos programas, su ciclo de consumo particular con respecto a otros géneros hasta 7 dias después, los subgéneros más vistos y la hora a la que se emitieron por la vía tradicional los contenidos más demandados luego en diferido. Para responder a los objetivos planteados se recopilaron los datos de los 10 programas más vistos en diferido durante 9 meses para alcanzar una muestra de 2.750 contenidos. Los resultados muestran que los programas infantiles destacan por el incremento de su audiencia tradicional tras la suma del diferido al tratarse mayoritariamente de productos que no caducan con el paso del tiempo y a cuyos telespectadores parece no importarles la fecha de emisión. De esta manera, observamos que los programas infantiles son los contenidos con un consumo más repartido a lo largo de los días y que los más vistos en diferido son los emitidos en lineal en los horarios de protección infantil en la televisión tradicional.

Palabras clave: Audiencia en diferido; Programas de televisión; Menores de edad; Vídeo bajo demanda; Cadenas infantiles; Consumo audiovisual

\section{Abstract}

This article presents one of the first studies focused on the time shifted consumption of children's television shows in Spain. The research analyzes the quantification of the audience's power in these programs, their particular consumption cycle concerning other genres until 7 days later, the most watched subgenres and the time when traditional content was broadcasted before his time shifted consumption. In order to respond to the objectives, the data of 10 most watched shows were collected for 9 months to reach a sample of 2,750. The results show that children's programs achieve a notable increase in their traditional audience after adding the time shifted audience, because it is mostly products that do not expire with the passage of time and whose viewers do not seem to care about the date of broadcast. In this way, we observe that children's shows are the content with a more distributed consumption 
throughout the sample and that the most watched time shifted contents are those broadcast firstly in the schedules of child protection for traditional television.

Key Words: Time shifted audience; Television shows; Minors; Video On Demand; Children's channels; Audiovisual consumption

\section{Resumo}

Este artigo apresenta um dos primeiros estudos focados no consumo diferido de programas de televisão infantis na Espanha. A pesquisa analisa quantificar o peso da audiência adiada nestes programas, seu ciclo particular de consumo em relação a outros gêneros até 7 dias, subgêneros mais vistos eo tempo que foram emitidos no conteúdo forma tradicional mais os réus então adiaram. Para responder aos objetivos propostos, os dados dos 10 programas mais vistos foram coletados em diferido por 9 meses para atingir uma amostra de 2.750 conteúdos. Os resultados mostram que programas infantis com destaque para o aumento de sua tradicional audiencia após a montante diferido ser principalmente de produtos que não expiram com $o$ passar do tempo e cujos espectadores parece não importa a data de emissão. Assim, nota-se que programas infantis estão contentes com mais consumo distribuídos por dias e atrasou o mais visto são os emitidos na proteção horários criança linear na televisão tradicional.

Palavras chave: Público diferido; Programas de televisão; Menores; Vídeo sob demanda; Cadeias infantis; Consumo audiovisual

\section{Introducción}

La programación infantil ha desaparecido de las cadenas generalistas. En los 90, con la llegada de las televisiones privadas a España, el contenido dirigido a los más pequeños era muy competitivo, pero se redujo paulatinamente durante el siglo XXI (Fernández, 2012). Según Digón (2008), el motivo fue la imposición del modelo de televisión comercial, que descubrió que al eliminar los contenidos infantiles muchos pasaban a ver programas para adultos. En 2003 el proyecto de investigación "La televisión y los niños: programación infantil y anomia televisiva" 


\section{ARTÍCULOS DE INVESTIGACIÓN}

ya revelaba que había caído en picado la emisión de programas para menores y que solo se les atendía en las primeras horas de la mañana (Cebrián, 2003). La calidad también había descendido con apenas producción propia al quedar relegada a programas contenedores como excusa para incorporar material extranjero (Pérez y Núñez, 2009). Vázquez (2009) afirma que parte de la pérdida de calidad de estos contenidos se debe a que la programación infantil sufre numerosas repeticiones y reposiciones (el 36,45\% tiene más de diez años de antigüedad e incluso hay un $3,45 \%$ con más de veinte años). Otra constante es la variabilidad, los programas se cambian o se eliminan con facilidad y sin previo aviso (Digón, 2008). Todo ello junto con la poca diversidad de horarios en los que los menores pueden ver sus programas preferidos provocó un trasvase de niños desde las cadenas generalistas a las temáticas (Pérez y Núñez, 2009). Es decir, “la tradicional idea de tener barras o franjas de programación por la mañana, y por la tarde, es una manera de programar que hoy día no responde a los cambios en los visionados infantiles" (Fuenzalida, 2007: 50).

Con la aparición de la Televisión Digital Terrestre (TDT) surgieron nuevos canales temáticos y los más pequeños se convirtieron en su público objetivo. Clan TV (RTVE), Disney Channel, Nickelodeon y Nick Jr (Viacom Inc.) y Boing (Mediaset) aparecieron en respuesta a la gran demanda que había en ese momento (Fernández, 2012; Fernández y Díaz-0campo, 2014). No obstante, en sus inicios, la TDT no trajo la diversidad de contenidos que prometía y se convirtió en un contenedor para sacar el contenido con el que ya contaban las grandes corporaciones (Fernández, 2012).

Pero con la llegada de las segundas pantallas (tabletas, móviles, etc.), en un primer momento, lejos de bajar la audiencia, se impulsó el consumo de televisión, aunque a diferencia de lo que sucede en otros países como Francia o Italia donde los más pequeños eligen las cadenas generalistas, los canales infantiles surgidos con la TDT son los preferidos por los menores en España (Díaz-Campo y Fernández, 2014). Según el informe Kids TV Report, de Eurodata TV Worldwide (2017), los niños europeos pasaron un tercio del tiempo que ven televisión en canales infantiles, llegando al $50 \%$ en edades preescolares. El desarrollo de sus marcas en plataformas de fácil acceso ha ayudado a confirmar el éxito de los canales especializados en programación infantil. Por ejemplo, en 2017 Clan TV fue el canal más visto entre 
el público de 4 a 12 años (15\% de share en esa franja de edad), seguido por Boing $(11,7 \%)$ y Disney Channel con un 9,8\% (Barlovento Comunicación, 2018).

\subsection{Los canales de televisión dirigidos al público infantil en España}

Clan TV es el canal temático infantil del grupo de televisión pública RTVE. El $50 \%$ de su programación va dirigida a un público de entre 3 a 6 años, mientras el otro $50 \%$ es para mayores de 7 años. Emite 24 horas de programación ininterrumpidas, los 7 días de la semana. En su parrilla encontramos más de 30 productos audiovisuales diferentes: un $63,33 \%$ son dibujos animados y un $20 \%$ series de ficción (Melgarejo y Rodríguez, 2011).

Los canales infantiles de la compañía Disney se centran en el entretenimiento, pero se complementa con contenido educativo, sobre todo en los canales que están dirigidos a un público más pequeño (Disney Junior, para los menores de 6 años). Disney Channel dispone de un $71,11 \%$ de contenidos destinados a un target mayor de 7 años y un 28,88\% para un público más infantil (de 4 a 6 años). Cuenta con 45 contenidos que pertenecen a los géneros de ficción $(44,44 \%)$, educativos $(15,55 \%)$ y musicales (4,44\%). En cuanto al formato más utilizado destacan los dibujos animados $(48,88 \%)$ y las series de ficción $(37,77 \%)$ de la programación de este canal (Melgarejo y Rodríguez, 2011). Por su parte, Boing es el canal temático infantil y juvenil de Mediaset, producido por Turner Broadcasting System, que es quien proporciona la mayor parte del contenido. El 57,1\% de los dibujos animados son estadounidenses, el $21,4 \%$ de Japón seguido por Francia (14,2\%) y Canadá (7,14\%) (Alonso, 2014).

Nickelodeon se dirige a los mayores de 6 años, el 91,30\% de sus contenidos atiende a un público de entre 7 a 12 años. Por ello, Viacom creó otro canal exclusivo para niños preescolares de 3 a 6 años (Nick Jr.) Nick Jr., tiene una programación muy escasa, 7 series de dibujos animados, de los que 5 de estos contenidos se dirigen al género educativo (Melgarejo y Rodríguez, 2011). También hay que tener en cuenta que los gustos de los niños de 9 y 10 años son más equilibrados entre los dibujos animados, los shows familiares y las comedias, aunque se aprecia una tendencia en las niñas a perder pronto el gusto por los dibujos animados (Garitaonandia, Juaristi y Oleaga, 1999). 
Pero la expansión de la televisión en diferido le ha dado a los más pequeños la posibilidad de contar con mayor flexibilidad y disfrutar del contenido en el momento que ellos quieran. Por eso, este artículo pretende analizar el comportamiento del consumo de los contenidos infantiles tras su emisión lineal en los canales de televisión tradicionales.

\subsection{Estudios sobre el consumo en diferido de contenido infantil}

El desarrollo de las nuevas tecnologías ha permitido que los usuarios puedan consumir los programas de televisión después de su emisión tradicional, con las televisiones conectadas o smart TV. La televisión conectada es el medio más utilizado para ver contenido infantil (34\%) seguido por las tabletas $(27 \%)$ y la televisión con disco duro con un 21\% (IAB, 2015). La denominada audiencia en diferido (o time shifted) es aquella que se crea a partir del primer minuto en el que un programa se emite por televisión, y ha empezado a medirse en España a partir de febrero de 2015 por la empresa Kantar Media (Optimedia, 2015). Waisman, Hidalgo y Rossi (2017: 186) afirman que “los dispositivos móviles presentan indudables atractivos: su portabilidad, la comodidad para los padres en cuanto a entretener a los niños mientras ellos realizan tareas necesarias, el acceso a innumerables fuentes de entretenimiento e información, y la disminución relativa de costos". La multiplicación de pantallas y de posibilidad de entretenimiento tecnológico (y no solo basado en el consumo de televisión en diferido) ha desembocado en que los niños se hagan cada vez más sedentarios y no puedan practicar habilidades como caminar y correr, y eso demora su desarrollo (Quirantes, 2019).

En cuando a la línea específica de investigación de la audiencia en diferido, Gallardo y Sierra (2017) concluyen que las cadenas temáticas españolas son las que adquieren mayor crecimiento porcentual (frente a las generalistas) de su consumo en diferido con respecto a su emisión lineal y que los canales temáticos con los programas más consumidos en diferido son los de ficción (45\%) seguidos de los infantiles $(20 \%)$, pero este estudio se limita a un mes de análisis de los 10 contenidos más vistos en diferido. Gallardo-Camacho y Lavín (2018) destacan la relevancia de los contenidos infantiles según el número de visualizaciones al otorgarle a estos programas un $26 \%$ de peso entre los 10 contenidos más vistos en un periodo de análisis más amplio. 
Otro estudio realizado en Reino Unido revela que ha aumentado el número de niños de 3 a 11 años que no ve la televisión en un televisor tradicional y que en 2016 creció a 119 minutos por semana el tiempo que los más pequeños dedican al contenido en diferido (Ofcom, 2017). Por su parte, Statista (2018) también desglosa el consumo de la audiencia en diferido en Reino Unido donde concluye que las series sobresalen con un $37 \%$, le siguen los documentales con un $26 \%$, el entretenimiento y el cine (22\%), la música (18\%), los contenidos infantiles (15\%), los deportes (14\%) y las noticias (5\%).

El televisor es la pantalla preferida en general para los espectadores de más de 6 años (91\%), seguido del ordenador (55\%), pero las tabletas son la gran revelación: en un año aumentó el uso entre los menores de entre 6 a 14 años en 10 puntos, llegando al 67\% (Mediametrie, 2018). Si nos centramos en el consumo de televisión en España, los datos de 2017 nos indican que los niños de 4 a 12 años dedican 145,5 minutos de media al día, de los que 141,6 son emisiones lineales, pero 3,9 minutos en diferido: lo que se traduce en un aumento en 0,9 minutos con respecto al año anterior (Barlovento Comunicación, 2018). Este crecimiento del consumo en diferido está generando una pérdida de espectadores que dejan de ver la televisión tradicional (Santago y González, 2015). De hecho, un estudio que analiza la audiencia en diferido en 62 países ya ha detectado un trasvase masivo de la audiencia a los nuevos servicios de televisión bajo demanda excepto cuando se emiten programas o eventos en directo en la televisión tradicional (Becker, Abreu, Nogueira y Cardoso, 2018).

Con respecto a los estudios de la audiencia en diferido más generales, González Neira y Quintas Froufe (2016) analizan el consumo de las series de ficción españolas y destacan la falta de relación con el éxito de la audiencia obtenida en su emisión lineal al igual que concluyen Gallardo-Camacho, Lavín y Fernández-García (2016). Por tanto, un contenido infantil podría triunfar en diferido, aunque pase desapercibido en su emisión lineal.

Además, nos encontramos ante un nuevo contexto en el que el consumo de contenidos audiovisuales infantiles también se produce en otros canales digitales: plataformas de vídeo por internet gratuitas (Youtube o Youtube Kids) o de pago 
como Netflix (en su configuración familiar). Aunque uno de los puntos fuertes de Netflix es su producción propia, su debilidad es que depende de los contenidos producidos por otras compañías (Allen, Feils y Disbrow, 2014). De hecho, es tal el interés por el mercado de los más pequeños que Disney ha retirado todo su catálogo de contenidos de Netflix para crear su propia plataforma llamada Disney + y con un precio más bajo.

Con respecto a este caso, Lotz (2018) asegura que hay mercado para las nuevas plataformas de vídeo de pago y concluye que convivirán porque cada una va dirigida hacia públicos diferentes (Disney, por ejemplo, va más hacia el público objetivo infantil que Netflix) pero la misma autora es más contundente al asegurar que ha llegado el fin de los paquetes de canales de televisión tradicionales temáticos que se contrataban en los hogares estadounidenses. Con la llegada de estas plataformas de pago denominadas 0TT (Over The Top) los telespectadores de menor edad han empezado a tener unos hábitos de consumo basados en la visualización de contenidos serializados en pequeños periodos de tiempo. Es lo que se denomina el fenómeno del Binge Watching: los atracones de series basados en un consumo masivo y continuado entre las capas más jóvenes (Matrix, 2014). Además, con la expansión de internet y de las aplicaciones móviles, los niños empiezan a empoderarse audiovisualmente ante la posibilidad de crear contenidos interactivos fácilmente (Monroy-Hernández y Resnick, 2008). Se produce la llegada de “los menores influyentes en internet" y Tur-Viñes, Núñez y González-Río (2018: 1211) piden precisamente mayor regulación para controlar la presencia de anunciantes en los vídeos que suben los niños al detectar "un estilo de gestión cuasiprofesional y la omnipresencia de marcas de manera simultánea". Sin embargo, en este artículo vamos a centrarnos en el consumo que hacen los niños sobre lo que se ha emitido previamente en la televisión lineal, aunque es relevante conocer el contexto en el que ahora consumen contenidos audiovisuales, hasta el punto de que Lewin (2010) ya advertía de que si "tu hijo está despierto, está conectado". Esa conexión nativa a internet justifica la relevancia de esta investigación al analizar el comportamiento del telespectador de menor edad.

Este artículo supone la culminación de una macro investigación centrada en uno de los primeros análisis de la audiencia en diferido en España. Sin embargo, como 
detallaremos en la metodología, en esta ocasión nos centraremos en la audiencia infantil para crear una investigación inédita en España donde se analiza el fenómeno del consumo en diferido de la televisión tradicional por los niños.

\subsection{Objetivos de la investigación}

Antes de desarrollar la metodología, planteamos los cuatro objetivos de la investigación necesarios para crear una de las primeras radiografías del consumo en diferido focalizado exclusivamente en los contenidos infantiles:

- 01: Analizar el peso de los programas infantiles más vistos en la audiencia en diferido con respecto al resto de programas.

- 02: Comparar el ciclo de consumo de los programas infantiles en su visionado en diferido con respecto al resto de contenidos.

- 03: Analizar los subgéneros infantiles que tienen más peso en el consumo en diferido.

- 04: Detectar a qué hora se emiten en lineal los contenidos infantiles que más se consumen después en diferido.

Con la consecución de estos objetivos ahondaremos en el análisis de la audiencia en diferido de los programas infantiles.

\section{Metodología}

Para poder dar respuesta a los objetivos, planteamos una metodología cuantitativa basada en la recolección y manipulación de datos de audiencia de los 10 programas de televisión con mayor audiencia en diferido cada día durante nueve meses (desde el 25 de marzo de 2016 hasta el 25 de diciembre de 2016). Los datos se han obtenido diariamente a partir del programa informático Infosys + (2016) de Kantar Media (la empresa que mide la audiencia tradicional y en diferido en España). Dentro de las dificultades para estudiar con exactitud las audiencias de televisión, nos decantamos 
por analizar los datos cuantitativos de la empresa que facilita la información aceptada por el mercado audiovisual en España. Consideramos además que el periodo de estudio es lo suficientemente amplio como para alcanzar resultados concluyentes, se trabaja con una muestra de 2.750 programas obtenidos durante 275 días (son 275 y no 276 porque hay un día de la muestra que no se pudo acceder a los datos por fallos en el sistema: 11 de diciembre de 2016). Al estar centrada esta investigación en los programas infantiles hay que tener en cuenta que la audiencia en España se mide a partir de los 4 años. Dentro del concepto programa infantil incluimos los siguientes subgéneros clasificados por Melgarejo y Rodríguez (2012): dibujos animados (o series de animación), series de ficción, largometraje (cine), magacines (se desarrollan en un plató de televisión con un presentador), concursos y talk shows dirigidos a menores y principalmente por debajo de los 14 años. De cada uno de los diez programas más vistos en la audiencia en diferido de cada día se extrajeron los siguientes datos: nombre del programa, fecha y hora de emisión, posición entre los diez más vistos, número de espectadores en su emisión lineal, número de espectadores en su emisión en diferido en todo su ciclo de consumo, su género y subgénero. Precisamente, para poder realizar esta investigación hemos recurrido a la clasificación de los géneros de televisión porque es la única forma de organizar los textos audiovisuales según una serie de rasgos distintivos y de propiedades textuales e intertextuales (Wolf, 1984). El propio Wolf es consciente de que la pertenencia a esos "clubes de géneros" no es rígida y por eso hemos creado nuestra propuesta de clasificación a partir de la realizada por otros autores (Barroso, 1996; Bustamante, 2008): géneros informativos (informativos o noticieros, magacines de actualidad, documentales, magacines deportivos, retransmisiones de eventos deportivos y no deportivos), géneros de ficción (series españolas, series extranjeras, cine, infantil) y los géneros de entretenimiento (reality shows, docu-realities, talent shows, humor, concursos y magacines de entretenimiento).

De cara a la metodología hay que considerar que la audiencia en diferido se publica una semana después de la emisión lineal y que tiene en cuenta la reproducción del contenido después del minuto siguiente a su hora de inicio hasta siete días después. Es decir, las audiencias en diferido analizadas en nuestra muestra se han difundido en su emisión lineal desde el 18 de marzo de 2016 hasta el 18 de diciembre de 2016. Kantar Media contabiliza la audiencia no lineal del mismo día 
de emisión (denominada VOSDAL, Viewing On The Same Day As Live) y la de los siete días posteriores. El sistema por el que se mide la audiencia no lineal se denomina audio-matching: el audímetro tiene un receptor con el que reconoce el audio de las emisiones e identifica el contenido a través del audio registrado en una base de datos (independientemente del método con el que el espectador consuma ese contenido: a través de un ordenador conectado al televisor, de un grabador digital, etc.). Por tanto, el sistema de Kantar Media no contabiliza lo que vemos en otros dispositivos diferentes al televisor, es decir, no se mide el fenómeno denominado por Gillan (2010) como placeshifting definido como un nuevo término que se refiere a la habilidad de la audiencia de trasladar el consumo de programas de televisión en sus dispositivos móviles. En este sentido, nos encontramos con limitaciones vinculadas al sistema de medición de audiencias en los que se basa esta investigación. Por eso, hay autores como Hernández-Pérez y Rodríguez (2016) que aseguran que se necesitan nuevas métricas para las audiencias centradas más en los usuarios que en los medios. Sin embargo, estimamos que estos datos están legitimados por los grandes grupos de comunicación y que las conclusiones que se obtengan a partir de ellos son determinantes para lograr los objetivos planteados.

El sistema de extracción de datos y la muestra de esta investigación han sido utilizadas previamente para la realización de otras investigaciones (Gallardo-Camacho y Lavín, 2018, 2019; Gallardo-Camacho, Sierra y Lavín, 2019) pero ninguna de ellas se centró en el ámbito infantil. Este artículo pretende unificar los hallazgos a partir de la misma muestra, pero centrándonos en el papel de los niños en el ámbito de la audiencia en diferido y así ofrecer información inédita. Para la elaboración de algunas Figuras y Tablas se recurre a los datos en bruto de esas investigaciones previas a los que han tenido acceso los autores. Pero también se aportan resultados elaborados expresamente para esta investigación: por ejemplo, se realiza la división por subgéneros infantiles y la clasificación según el horario de emisión para responder a los objetivos planteados.

\section{Resultados}

Tras el planteamiento metodológico, pasamos a mostrar los resultados de la investigación en relación con los objetivos planteados. 


\section{ARTÍCULOS DE INVESTIGACIÓN}

\subsection{El peso de los programas infantiles en diferido}

En primer lugar, para cumplir el 01 hay que tener en cuenta que nos enfrentamos a una muestra de $\mathrm{N}=2750$ obtenida de los 10 programas más vistos en diferido en España durante 9 meses. Por ello, es relevante saber cuántos de esos programas se encuentran bajo la denominación de infantiles (Figura 1).

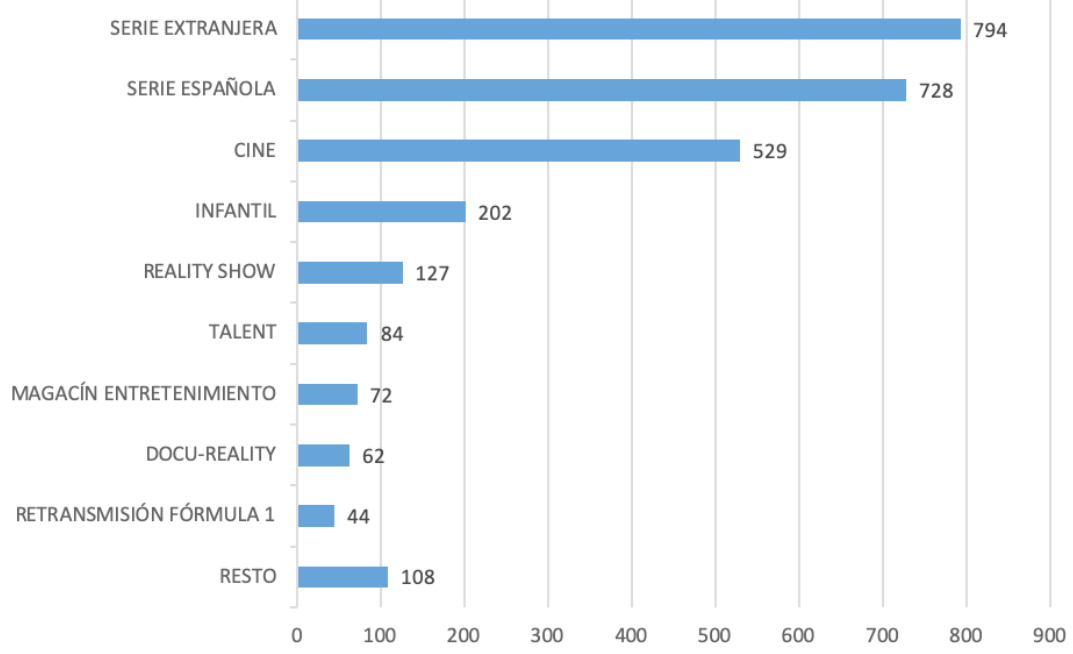

Figura 1: Frecuencia de aparición de los contenidos infantiles más vistos en diferido frente al resto de géneros $(N=2750)$. Fuente: elaboración propia a partir de datos de Gallardo-Camacho y Lavín (2019).

De esta manera, observamos en la Figura 1 que los contenidos de temática infantil son los cuartos más vistos con una frecuencia de 202 programas frente a las series extranjeras (794), las series españolas (728) y las películas (529). Por tanto, se puede determinar que esa cuarta posición es relevante si tenemos en cuenta el gran número de géneros y subgéneros captados en la muestra como hemos detallado en la metodología. $\mathrm{Si}$ aglutinamos todos los subgéneros en una clasificación más amplia podremos observar el peso real de los contenidos infantiles con respecto a toda la muestra (Figura 2). 


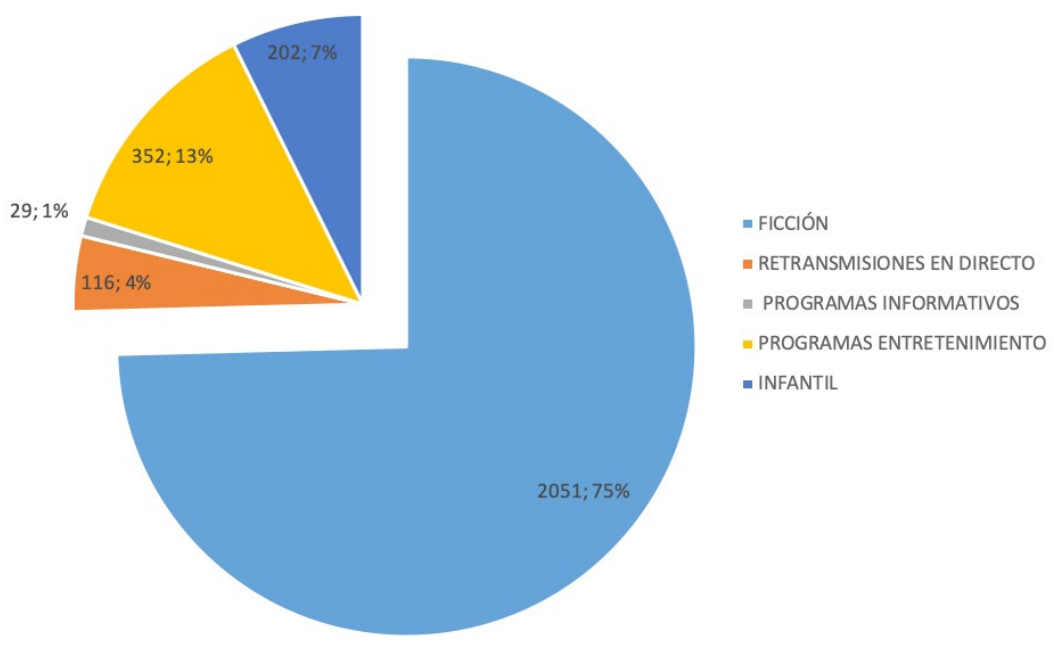

Figura 2: Porcentaje de los géneros más consumidos en diferido ante los programas infantiles.

Fuente: elaboración propia a partir de datos de Gallardo-Camacho y Lavín (2019).

La ficción reina el consumo en diferido con un $75 \%$ de toda la muestra, seguida de los programas de entretenimiento (13\%), los contenidos infantiles ( $7 \%$ con los 202 programas que vimos en la Figura 1) y, por último, los programas de actualidad con un $5 \%$ ( $1 \%$ de los informativos y $4 \%$ de las retransmisiones en directo). Pero para determinar también el peso de los contenidos infantiles en diferido hay que observar cuánto crecen tras su emisión lineal (Figura 3).

En la Figura 3, observamos que los contenidos infantiles destacan ante la mayoría de los géneros, al casi duplicar su audiencia lineal con un incremento de un $+92 \%$ de su audiencia tradicional tras sumarle los espectadores acumulados durante ocho días (el día de emisión y los siete posteriores). Solo se produce un incremento más destacable de telespectadores acumulados en diferido en el caso de las series extranjeras (+230\%) y de las películas $(+141 \%)$. 
168 | Jorge Gallardo-Camacho, Eva Lavín y Javier Sierra Sánchez

\section{ARTÍCULOS DE INVESTIGACIÓN}

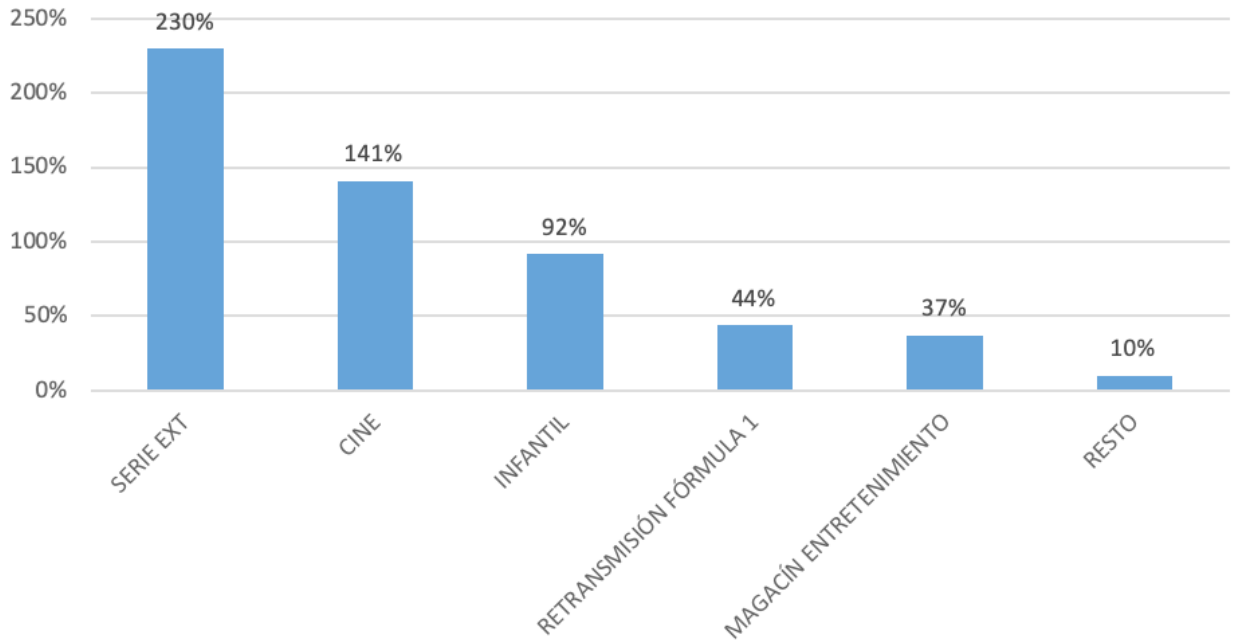

Figura 3: Incremento de la audiencia lineal tras acumular la audiencia en diferido por géneros. Fuente: elaboración propia a partir de datos de (Gallardo-Camacho y Lavín, 2019).

En la Figura 3, observamos que los contenidos infantiles destacan ante la mayoría de los géneros, al casi duplicar su audiencia lineal con un incremento de un $+92 \%$ de su audiencia tradicional tras sumarle los espectadores acumulados durante ocho días (el día de emisión y los siete posteriores). Solo se produce un incremento más destacable de telespectadores acumulados en diferido en el caso de las series extranjeras $(+230 \%)$ y de las películas $(+141 \%)$.

\subsection{El ciclo de consumo de los programas infantiles en diferido}

En cuanto a la 02, vamos a analizar el ciclo de consumo de los programas infantiles en su visionado en diferido con respecto al resto de contenidos. De esta manera, en la Figura 3 hemos visto cuánto crecen los espectadores de los programas, pero ahora es relevante determinar cuándo crecen a lo largo de los días de medición (Figura 4). 
Los programas infantiles de televisión y su consumo en diferido en España | 169 ARTíCULOS DE INVESTIGACIÓN

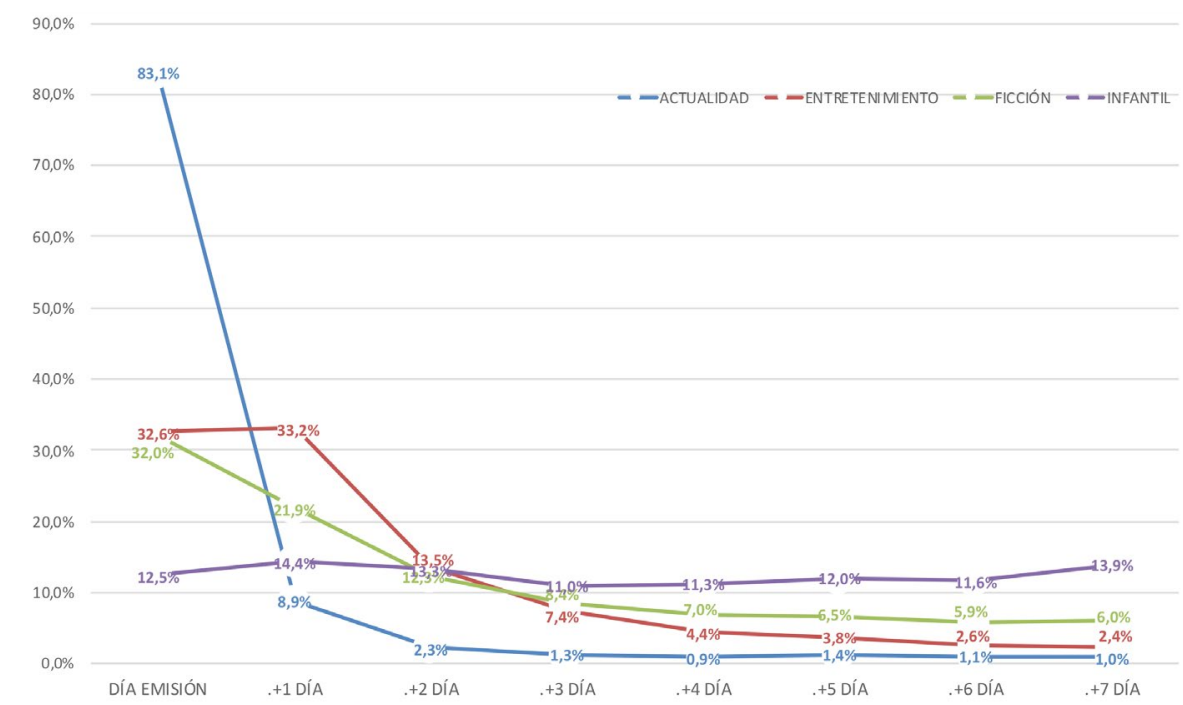

Figura 4: El ciclo de consumo en diferido de los programas infantiles ante el resto de géneros.

Fuente: elaboración propia a partir de datos de Gallardo-Camacho, Sierra y Lavín (2019).

En la Figura 4, observamos que el consumo en diferido de los programas infantiles presenta un comportamiento atípico con respecto al resto de géneros. Por un lado, los programas de actualidad acumulan masivamente su audiencia en diferido el mismo día de emisión (VOSDAL) y a partir del primer día posterior se produce una gran bajada en su consumo. En el caso de los programas de entretenimiento, los espectadores que ven el programa después de su emisión lineal se reparten más entre los primeros cuatro días $(32,6 \%$ VOSDAL, 33,3\%+1, 13,5\% + y 7,4\%+3). Por su parte, ya hemos visto que la ficción lidera el peso del consumo en diferido (Figuras 1 y 2 ) pero presenta un desgaste del consumo a medida que pasan los días produciéndose una acumulación del $66,2 \%$ de la audiencia en diferido los tres primeros días (VOSDAL, $+1 \mathrm{y}+2$ ). Pero centrándonos en nuestro objeto de estudio, el consumo en diferido de los programas infantiles no se ve afectado por el paso de los días y tiene un ciclo de consumo estable durante todos los días de análisis. El día de emisión (VOSDAL) acumula un $12,5 \%$ de su audiencia en diferido y el último presenta un $13,9 \%(+7)$ encontrándonos con el $14,4 \%$ como el dato más alto $(+1)$ y con un $11 \%$ como el resultado más bajo (+3). Es decir, los programas infantiles tienen un ciclo de consumo repartido y estable durante los días que analiza Kantar Media. 
170 | Jorge Gallardo-Camacho, Eva Lavín y Javier Sierra Sánchez

\section{ARTÍCULOS DE INVESTIGACIÓN}

\subsection{Los subgéneros infantiles con más peso en la audiencia en diferido}

En cuanto a la clasificación de los subgéneros infantiles más consumidos en diferido (03), hemos tenido en cuenta la clasificación de Melgarejo y Rodríguez (2012) tal y como hemos advertido en la metodología.

\begin{tabular}{|l|c|c|c|c|}
\hline \multicolumn{1}{|c|}{ Canales } & Dibujos & Serie & Cine & Magacines \\
\hline Clan & 132 & 0 & 0 & 0 \\
\hline Disney Channel & 7 & 17 & 5 & 1 \\
\hline Disney Junior & 13 & 0 & 0 & 0 \\
\hline Boing & 3 & 0 & 0 & 0 \\
\hline Nickelodeon & 1 & 0 & 0 & 0 \\
\hline Nickelodeon Jr & 22 & 0 & 17 & 0 \\
\hline Antena 3 & 178 & 0 & 6 & 0 \\
\hline T0TAL & 0 & 17 & 0 & 0 \\
\hline
\end{tabular}

Tabla 1: Los subgéneros de los programas infantiles más vistos en diferido.

Fuente: elaboración propia.

En la Tabla 1 establecemos una clasificación de los subgéneros infantiles pero, antes de todo, hay que tener en cuenta que los programas que aparecen en la muestra proceden solo de 6 cadenas de televisión temáticas: Clan TV, Disney Channel, Disney Junior, Boing, Nickelodeon y Nickelodeon Jr; y de una generalista (Antena 3). De esta manera, observamos que los dibujos animados o series de animación son los contenidos infantiles más vistos en diferido $(88,1 \%, 178)$, seguidos muy de lejos por las series de ficción $(8,4 \%$, $17)$, el cine $(3 \%, 6)$ y los magacines con solo 1 programa (los concursos y talk shows no 
obtuvieron representación). Si vemos el detalle de las 202 emisiones de dibujos más vistas, son sólo 30 los programas que aparecen. "La patrulla canina” es el programa que aparece más veces en la muestra con 56 capítulos, seguido por "Peppa Pig" (33), “Juan y Tolola" (13), "Los superminihéroes" (14). Las series más vistas se concentran en dos: "Soy Luna" (9) y “Campamento Kikiwaka" (8), las dos de Disney Channel.

Para comprender mejor el fenómeno de los programas infantiles más consumidos en diferido es necesario observar la Tabla 2.

\begin{tabular}{|c|c|c|c|c|c|}
\hline Cadena & Horario & Programa & $\begin{array}{c}\text { Diferido } \\
\text { (miles) }\end{array}$ & $\begin{array}{l}\text { Lineal } \\
\text { (miles) }\end{array}$ & $\%$ por diferido \\
\hline CLAN & Tarde & La patrulla canina & 284 & 193 & $147 \%$ \\
\hline CLAN & Mañana & La patrulla canina & 171 & 250 & $68 \%$ \\
\hline CLAN & Tarde & La patrulla canina & 146 & 270 & $54 \%$ \\
\hline CLAN & Tarde & La patrulla canina & 143 & 215 & $67 \%$ \\
\hline $\begin{array}{l}\text { DISNEY } \\
\text { CHANNEL }\end{array}$ & Mañana & $\begin{array}{l}\text { Prodigiosa: las aventuras de } \\
\text { Ladybug }\end{array}$ & 130 & 276 & $47 \%$ \\
\hline CLAN & Mañana & La patrulla canina & 127 & 189 & $67 \%$ \\
\hline CLAN & Tarde & La patrulla canina & 126 & 337 & $37 \%$ \\
\hline CLAN & Tarde & La patrulla canina & 117 & 224 & $52 \%$ \\
\hline CLAN & Tarde & La patrulla canina & 115 & 197 & $58 \%$ \\
\hline CLAN & Sobremesa & La patrulla canina & 112 & 408 & $27 \%$ \\
\hline CLAN & Sobremesa & La patrulla canina & 107 & 236 & $45 \%$ \\
\hline CLAN & Mañana & La patrulla canina & 103 & 236 & $44 \%$ \\
\hline CLAN & Sobremesa & La patrulla canina & 103 & 304 & $34 \%$ \\
\hline $\begin{array}{l}\text { DISNEY } \\
\text { CHANNEL }\end{array}$ & Mañana & $\begin{array}{l}\text { Prodigiosa: las aventuras de } \\
\text { Ladybug }\end{array}$ & 101 & 93 & $109 \%$ \\
\hline CLAN & Tarde & Peppa Pig & 100 & 181 & $55 \%$ \\
\hline
\end{tabular}

Tabla 2: Los 15 programas infantiles más vistos en diferido de la muestra.

Fuente: elaboración propia. 
En la Tabla 2 observamos que el programa infantil más visto de la muestra es "La patrulla canina" con 284.000 espectadores acumulados durante los 8 días que analiza Kantar Media frente a los 193.000 que vieron estos dibujos animados en su emisión lineal. Los 15 programas infantiles más vistos en diferido de la muestra promedian 132.000 telespectadores tras su emisión lineal, una cifra cuantitativamente relevante en cuanto a consumo audiovisual.

\subsection{La relación del horario de emisión lineal y los contenidos más vistos en diferido}

En cuanto al último objetivo de la investigación (04), es relevante saber a qué hora se emitieron los programas infantiles más vistos en diferido (Figura 5).

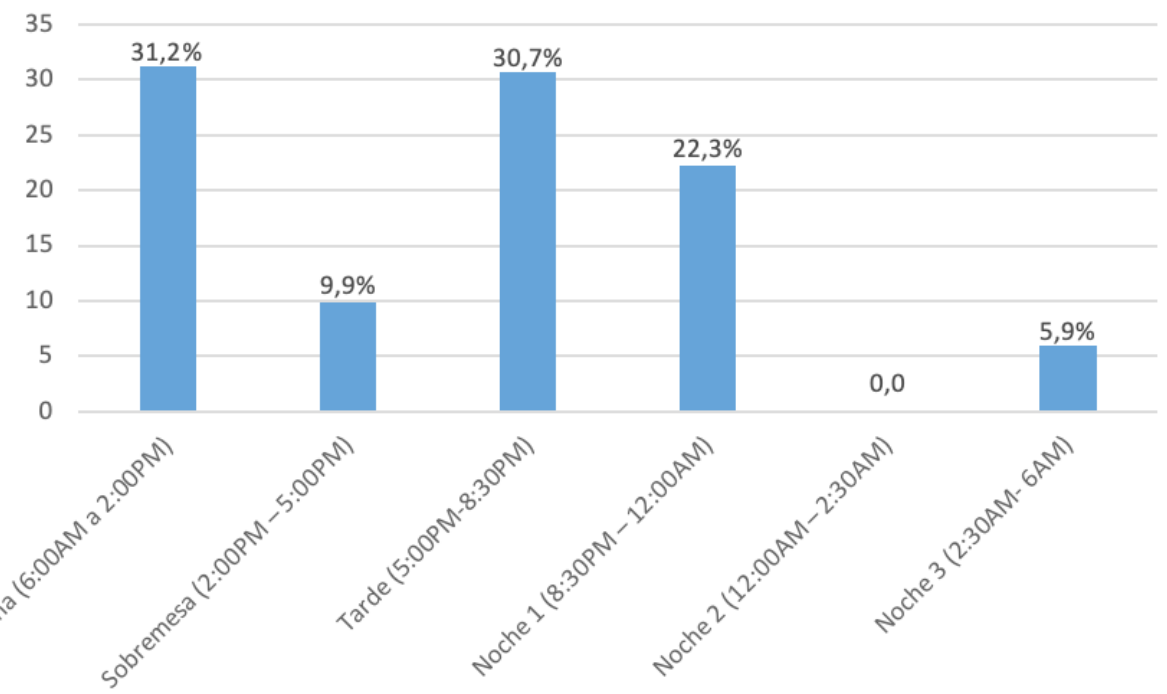

Figura 5: Hora de emisión lineal de los programas infantiles más vistos en diferido. Fuente: elaboración propia.

Si seguimos la organización de las franjas horarias de Kantar +Media, vemos en la Figura 5 que los programas más vistos en diferido son los que se emitieron previamente en las franjas de mañana $(31,2 \%$ de 6 AM a $2 \mathrm{PM})$ y tarde $(30,7 \%$ de $5 \mathrm{PM}$ a 8:30 PM). La franja de la noche también es destacable con un 22,3\% del consumo siendo inexistente la franja de la Noche 2 y más reducida la de la Noche 
$3(5,9 \%)$. Se presupone que el consumo de estos programas en todas las franjas donde hay presencia de niños se produce primordialmente en las primeras horas de cada intervalo (por ejemplo, de 6 a 8 AM en la franja de mañana): bien porque los niños han de ir al colegio en días laborables o porque ya es demasiado tarde como para no estar en la cama.

\section{Discusión y conclusiones}

Una vez expuestos los resultados, exponemos la discusión y conclusiones relacionadas con cada uno de los objetivos planteados.

En primer lugar, con respecto al 01, el peso de los programas infantiles es relevante en el consumo en diferido a pesar de que representen solo un $7 \%$ de los contenidos más vistos. Hay que tener en cuenta varios aspectos: por un lado, que en este trabajo solo se incluyen los 10 contenidos de todo tipo más vistos en toda la muestra (quedando fuera el resto de programas) y es meritorio que este tipo de programas tan especializados hayan entrado en la muestra con una frecuencia de 202 frente a una muestra de $\mathrm{N}=2750$; y por otro lado, que el peso de la audiencia en diferido es relevante en tanto en cuanto los programas infantiles crecen un $92 \%$ (Figura 3) gracias a los espectadores acumulados durante ocho días, una cifra muy superior a la de todos los subgéneros menos en el caso de las series extranjeras y el cine.

En cuanto al 02, podemos concluir que el paso de los días no es determinante en los contenidos infantiles para influir en su ciclo de consumo diferido ya que se reparte a lo largo de todo el periodo de análisis. De hecho, esta conclusión pone en tela de juicio la limitación que hace Kantar Media de medir a +7 días la audiencia no lineal. Quizás habría que ampliar este periodo, aunque entendemos que es el estándar establecido en la mayoría de los países del mundo (Optimedia, 2015).

Por su parte, también concluimos que los dibujos o series de animación se han convertido en el producto estrella más consumido en diferido (03) con un 88,1\% de representación de entre los 10 programas infantiles más vistos durante 9 meses. Que los contenidos infantiles sean mayoritariamente de ficción es lo que justifica 
en parte que destaque en el consumo en diferido ya que la ficción tiende a la mayor acumulación de espectadores (Madinaveitia y Merchante, 2015) y porque, en estos momentos, existe un incremento sostenido del consumo de ficción en internet (Lacalle y Gómez, 2017).

Por último, con relación al 04, concluimos que los programas infantiles más vistos en diferido son los que se emiten en las franjas donde hay más consumo potencial de contenidos infantiles. De hecho, la mañana y la tarde son las franjas que se corresponden con el horario de protección reforzada del menor en televisión según la Ley General de la Comunicación Audiovisual en España (BOE, 2010) situada entre las 8 y 9 horas de la mañana y entre las 17 y las 20 horas de la tarde en los días laborables. Si revisamos las parrillas de la cadena con más peso en la muestra (Clan TV) observamos que en su horario de madrugada no emite los contenidos estrella más vistos linealmente, sino que ofrece contenidos menos conocidos y con menos popularidad entre sus espectadores (ya que se presupone que están durmiendo). Por lo que, podemos concluir que estos resultados demuestran que el espectador de contenidos infantiles quiere ver lo que se emite en sus franjas preferidas de consumo tradicional.

Ante todo lo expuesto, concluimos que el consumo de programas infantiles en diferido podría seguir creciendo: por un lado, porque los niños tendrán más facilidades de acceso al consumo en diferido de lo que se emite en televisión (tanto por el acceso tecnológico en el hogar como por las facilidades que generen las propias cadenas) y, por otro lado, porque en estos datos de análisis aún no se mide el consumo en tabletas y teléfonos inteligentes. Es decir, la medición de audiencia en diferido no tiene en cuenta, por el momento, lo que pasa en dispositivos móviles que son muy usados por los niños para consumir también televisión en diferido.

En cuanto a otra posible limitación de la investigación, los datos siguen siendo representativos a pesar de haber sido captados en el año 2016 al tratarse de una muestra amplia ( $\mathrm{N}=2750)$ y captada en un periodo también relevante (9 meses de observación). A esto hay que sumar, que el sistema de medición de audiencia en diferido de Kantar Media sigue siendo el mismo (algo que podría variar a medio plazo y modificaría la interpretación de estos datos). 
Por su parte, es importante tener en cuenta la reflexión de Waisman, Hidalgo y Rossi (2017: 186) cuando concluyen que “los niños, para bien o para mal, tienen acceso a todo tipo de pantallas a edades cada vez más tempranas, $\mathrm{y}$, aunque pareciera que esta tendencia es inexorable, aún no están claras las ventajas y las desventajas de este contacto anticipado". Y es que el consumo televisivo está cambiando a un ritmo muy acelerado y es el comportamiento de los más pequeños de casa lo que va a marcar lo que suceda en los próximos años y a largo plazo en el mercado audiovisual.

\section{Referencias}

Allen, G., Feils, D. y Disbrow, H. (2014). The Rise and Fall of Netflix: what happened and where will it go from here? Journal of the International Academy for Case Studies, vol. 20, n² 2, pp. 119-128. URL: https://bit.ly/2Zb2KOX

Alonso, C. (2014). Análisis de los dibujos animados emitidos en televisión: personajes, estilos y mensajes (tesis doctoral)s. Universidad de Granada, España. URL: https://goo.gl/44BShD

Barlovento comunicación (2018). Análisis televisivo 2017. URL: https://goo.gl/ b9CRgm

Becker, V., Abreu, J., Nogueira, J. y Cardoso, B. (2018). 0 desenvolvimento da TV não linear e a desprogramação da grelha. Observatorio (OBS*) Journal, 2018, 199-216. doi: 10.15847/obs0BS12120181007

BOE (2010). Ley 7/2010 de 31 de marzo, General de la Comunicación Audiovisual. URL: https://goo.gl/1DbLYL

Cebrián, M. (2003). Contenidos infantiles en televisión. Nueva técnica analítica Global. Zer - Revista de Estudios de Comunicación, 8 (15). URL: https://goo. gl/H9AGgt

Díaz-Campo, J. y Fernández, E. (2014). Análisis de los perfiles en Facebook de los canales temáticos infantiles de carácter privado. En Actas Icono 14, pp. 273289. Madrid: España. URL: https://goo.gl/YN21gD

Digón, P. (2008). Programación infantil y TV sensacionalista: entretener, desinformar, deseducar. Comunicar, 31(XVI), pp. 65-76. doi: 10.3916/c31-2008-01-008

Eurodata TV Worldwide (2017). Kids TV Report: international kids TV consumption and hits. URL: https://goo.gl/8gxkRu 
176 | Jorge Gallardo-Camacho, Eva Lavín y Javier Sierra Sánchez

\section{ARTÍCULOS DE INVESTIGACIÓN}

Fernández, E. (2012). La programación infantil y juvenil de la televisión pública española: ¿oferta generalista o temática? El paso de La 2 a Clan TVE. Estudios sobre el mensaje periodístico, 18, pp. 313-323. doi: 10.5209/rev_ESMP.2012. v18.40985

Fernández, E. y Díaz-Campo, J. (2014). Los canales temáticos infantiles y juveniles en Facebook: análisis de los perfiles de Disney Channel, Boing y Neox. Comunicación y hombre, 10, pp. 179-194. URL: https://goo.gl/KuYo5x

Fuenzalida, V. (2007). Cambios en la relación de los niños con la televisión. Comunicar, 30, x. XV, pp. 49-54. URL: https://bit.ly/2InEtjk

Gallardo-Camacho, J., Lavín, E. y Fernández-García, P. (2016). Los programas de televisión deportivos y su relación con la audiencia social en Twitter en España. Revista Latina de Comunicación Social, 71, pp. 272-286. doi: 10.4185/ RLCS-2016-1095.

Gallardo-Camacho, J. y Sierra Sánchez, J. (2017). La importancia de la audiencia en diferido en el reparto del poder entre las cadenas generalistas y temáticas en España. Prisma Social, 18, pp. 173-191. URL: https://goo.gl/hVNz3H

Gallardo-Camacho, J. y Lavín, E. (2018). La importancia de la audiencia en diferido frente a la audiencia lineal en la televisión en España. Revista Observatorio OBS, 12, n4, pp. 140 - 158. doi: 10.15847/obs0BS12420181295

Gallardo-Camacho, J. y Lavín, E. (2019). Los géneros televisivos y la audiencia en diferido en España: la ficción vence a la actualidad, Estudios del Mensaje Periodístico, 2, 2019. [Aprobado y pendiente de ser publicado]. URL: https://goo.gl/18LLLG

Gallardo-Camacho, J., Sierra, J. y Lavín, E. (2019). El ciclo de consumo en diferido de los programas de televisión tras su emisión lineal. Comunicación y Sociedad, 32 (2), pp. 29-43. doi: 10.15581/003.32.2.29-43

Garitaonandia, C., Juaristi, P., y Oleaga, J. (1999). Qué ven y cómo juegan los niños españoles. Zer - Revista de Estudios de Comunicación, 4(6). URL: https://goo. gl/MUz5tf

Gillan, J. (2010). Television and New Media: Must-Click TV. New York: Routledge.

González Neira, A. y Quintas Froufe, N. (2016). El comportamiento de la audiencia lineal, social y en diferido de las series de ficción española. Revista de la Asociación Española de Investigación de la Comunicación, v. 3, n. 6, 27-33. URL: https://goo.gl/PzYfkf 
Hernández-Pérez, T. y Rodríguez Mateos, D. (2016). Medición integral de las audiencias: sobre los cambios en el consumo de información y la necesidad de nuevas métricas en medios digitales. Hipertext.net, núm. 14, pp. 1-14. doi: 10.2436/20.8050.01.32

IAB (2015). III Estudio Anual IAB Spain. TV Conectada y Video Online 2015. URL: https://goo.gl/Pr1e49

InfoSys (2016, i+). InfoSys, (versión i+). [Programa de ordenador].

Lacalle, C. y Gómez, B. (2017). La recepción televisiva española en la era multipantalla. Comunicación y Sociedad (México), 30, pp. 197 - 216. URL: https://goo.gl/NZyt8v

Lewin, T. (2010). If your kids are awake, there're probably online. The New York Times, 20 de Enero. URL: https://bit.ly/2UzRE7n

Lotz, A. (2018). When envisioning the future of TV, think of a shopping mall. The Conversation, Art and Culture. URL: https://bit.ly/2UzNmNj

Madinaveitia, E. y Merchante, M. (2015). Medición de audiencias: desafío y complejidad en el entorno digital. Harvard Deusto Marketing y Ventas, 131, 26 - 33. URL: https://goo.gl/9M9V1V

Matrix, S. (2014). The Netflix Effect: Teens, Binge Watching, and On-Demand Digital Media Trends. Jeunesse: Young People, Texts, Cultures, vol. 6, n 1, pp. 119-138. doi: 10.1353/jeu.2014.0002

Mediametrie (2018). More than half of web users have enriched their TV experience via another screen. URL: https://goo.gl/xgVP69

Melgarejo, I. y Rodríguez, M.M. (2011). Los canales infantiles politemáticos de televisión digital en España: análisis y clasificación. Doxa.comunicación, 120, pp.44-64. URL: https://goo.gl/5zmVH7

Melgarejo, I. y Rodríguez, M.M. (2012). Géneros y formatos en los canales infantiles politemáticos de televisión. Revista de Comunicación Vivat Academia, 13, pp.171-191. doi: 10.15178/va.2012.120.44-64

Monroy-Hernández, A. y Resnick, M. (2008). Empowering kids to create and share programmable media, Interactions - Pencils before pixels: a primer in handgenerated sketching, vol. 15, nº. 2, pp. 50-53. doi: 10.1145/1340961.1340974 Nielsen (2017). Our vision for the next 5 years. URL: https://goo.gl/q5ME3Z Ofcom (2017). Children and Parents: Media Use and Attitudes Report. URL: https:// goo.gl/qYBYho 
Optimedia (2015). Audiencia en diferido-Timeshift. URL: https://goo.gl/eG7w2z Pérez Ornia, J.R. y Núñez Ladevéze, L. (2009). Programación infantil en la televisión española. Inadecuada relación entre oferta y demanda. Telos: Cuadernos de comunicación e innovación, 54. URL: https://goo.gl/GJc3Hq

Quirantes, A. (2019). Las pantallas y el desarrollo de los niños pequeños. Cubahora, $\mathrm{n}^{0}$ 18, febrero. URL: https://bit.ly/2Nbd3wK

Santago, F. y González, I. (2015). "Nuevos tiempos y nuevos usos del tiempo libre en torno al consumo audiovisual". En: 310 Seminario de televisión Aedemo. Sevilla, 12 de febrero. URL: https://goo.gl/MFyDFC

Statista (2018). Share of television viewing time-shifted in the United Kingdom (UK) in 2016 and 2017, by genre. URL: https://goo.gl/npAkNc

Tur-Viñes, V., Núñez, P. y González-Río, M. (2018). Menores influyentes en YouTube. Revista Latina de Comunicación Social, 73, pp. 1211-1230. doi: 10.4185/RLCS2018-1303

Vázquez, T. (2009). Evaluación de la calidad de la programación infantil de las televisiones generalistas españolas. Revista Latina de Comunicación Social, 64, pp. 844 a 861. doi: 10.4185/RLCS-64-2009-866-844-861

Waisman, I., Hidalgo, E. y Rossi, M. (2017). Uso de pantallas en niños pequeños en una ciudad de Argentina. Arch Argent Pediatr, 116 (2), pp. 186-195. doi: 10.5546/aap.2018.e186

Wolf, Mauro (1984). Géneros y Televisión. Anàlisi, 9, pp. 189-198. URL: https:// goo.gl/wUZFzk

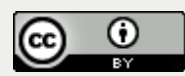

